Poincare Journal of Analysis E Applications

Vol. 2015 (2), Special Issue (IWWFA-II, Delhi), 129-137

(C) Poincare Publishers

\title{
ON PERTURBATION OF LOCAL ATOMS FOR SUBSPACES
}

\author{
Deepshikha and L.K. Vashisht ${ }^{\dagger}$ \\ Date of Receiving : : 02.05. 2015 \\ Date of Revision : : 29.07. 2015 \\ Date of Acceptance : 04.10 .2015
}

\begin{abstract}
A family of local atoms is a collection of vectors which are analysis and synthesis systems with frame-like properties for closed subspaces of a separable Hilbert space $\mathcal{H}$. In this paper, we present some perturbation results for local atoms in a subspace of a Hilbert space. Some algebraic properties of one of derivatives of local atoms are given.
\end{abstract}

\section{Background}

Frames in a Hilbert space are a redundant system of vectors which provides a series representation for each vector in the space. Duffin and Schaeffer [10] in 1952, introduced frames for Hilbert spaces, in the context of nonharmonic Fourier series. Let $\mathcal{H}$ be an infinite dimensional separable real (or complex) Hilbert space with inner product $\langle. .$.$\rangle linear in first entry. A countable sequence \left\{f_{k}\right\} \subset \mathcal{H}$ is called a frame (or Hilbert frame) for $\mathcal{H}$ if there exist numbers $0<m_{0} \leq M_{0}<\infty$ such that

$$
m_{o}\|f\|^{2} \leq \sum_{k}\left|\left\langle f, f_{k}\right\rangle\right|^{2} \leq M_{o}\|f\|^{2} \text { for all } f \in \mathcal{H} .
$$

The numbers $m_{0}$ and $M_{0}$ are called lower and upper frame bounds, respectively. They are not unique. If it is possible to choose $m_{o}=M_{o}$, then the frame $\left\{f_{k}\right\}$ is called Parseval frame (or tight frame). If the upper inequality in (1.1) satisfied, then we say that $\left\{f_{k}\right\}$ is a Bessel sequence in $\mathcal{H}$. Frames were revived by Daubechies, Grossmann and Meyer in [9]. For the utility of frames in different directions in applied mathematics an interested may refer to $[2,6]$.

Let $\left\{f_{k}\right\}$ be a frame for $\mathcal{H}$. The operator $S: \mathcal{H} \rightarrow \mathcal{H}$ given by

$$
S f=\sum_{k}\left\langle f, f_{k}\right\rangle f_{k}
$$

2010 Mathematics Subject Classification. 42C15, 42C30, 42C05, 46B15.

Key words and phrases. Hilbert frame, perturbation, local atoms, K-frames.

The first author is supported by CSIR, India vide File No.: 09/045(1352)/2014-EMR-I.

Lalit K. Vashisht is partly supported by R \& D Doctoral Research Programme, University of Delhi, Delhi-110007, India. Grant No. : RC/2014/6820.

Communicated by. Laura Gavruta

${ }^{\dagger}$ Corresponding author. 